\title{
Original Research \\ Development of patient-centered outcomes for labour and birth: a qualitative study
}

\author{
Geoffrey E. Johnson ${ }^{1}{ }^{\oplus}$, Lauren Kan ${ }^{2}$, Jennifer Nguyen $^{3}$, Kim Campbell ${ }^{3}$, Laura Ralph ${ }^{1}$,

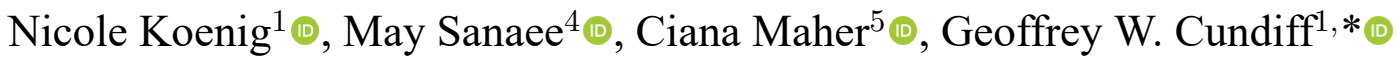 \\ ${ }^{1}$ Department of Obstetrics and Gynecology, University of British Columbia, Vancouver, BC V6Z 2K8, Canada \\ ${ }^{2}$ Department of Family Practice, University of British Columbia, Vancouver, BC V6Z 2K8, Canada \\ ${ }^{3}$ Division of Midwifery, Department of Family Medicine, University of British Columbia, Vancouver, BC V6Z 2K8, Canada \\ ${ }^{4}$ Department of Obstetrics and Gynaecology, University of Alberta, Edmonton, AB T6G 2R3, Canada \\ ${ }^{5}$ Women's Health Research Institute, University of British Columbia, Faculty of Medicine, Vancouver, BC V6Z 2K8, Canada \\ *Correspondence: geoff.cundiff@ubc.ca (Geoffrey W. Cundiff) \\ Academic Editor: Stefano Manodoro \\ Submitted: 25 October 2021 Revised: 14 December 2021 Accepted: 15 December 2021 Published: 14 January 2022
}

\begin{abstract}
Background: Current quality improvement models in obstetrics focus on prevention of adverse perinatal outcomes. The development of these metrics was based on expert opinion that did not account for patients' values. The ultimate aim of our research is to develop performance indicators for labour and birth that reflect the patient perspective. Methods: A qualitative interview design was used to engage a convenience sample, of recent ( $<1$ year) postpartum patients, in semi-structured interviews, where they shared their experiences of their recent birth. Patients were also asked to assess descriptions of adverse perinatal outcomes for readability and comprehension, towards developing accurate unbiased descriptions for a subsequent survey of patients to weight complications. Responses were recorded, transcribed, coded and analyzed using thematic analysis. thematic analysis. Results: Five themes emerged during the analysis: (1) desire for patient-centred care, (2) improved communication, (3) labour/birth, expectations and outcomes, (4) care team support during labour and birth, (5) continuing emotional and physical postpartum care. Conclusions: Patient-centred care and good health outcomes were the major values expressed by the patients in this study. Good communication and shared decision making led to patients describing their labour and birth as a satisfying experience. This study lays the foundation for developing a quality tool to measure the outcomes of birth and adverse outcomes from the patients' perspective.
\end{abstract}

Keywords: Performance indicators; Adverse perinatal outcome; Patient centered care; Labour outcomes; Shared decision making; Patient oriented research

\section{Key points}

Women seeking maternity care value patient-centred care and good health outcomes. They described birth as a satisfying experience when it included good communication and shared decision making. This study lays the foundation for developing a quality tool to measure the outcomes of birth and adverse outcomes from the patients' perspective.

\section{Introduction}

Hospitals engage in quality improvement (QI) activities in order to mitigate deficiencies in the quality of care based on objective monitoring of outcomes. The Institute of Medicine (IOM) describes quality care as safe, effective, patient-centered, timely, efficient, and equitable [1]. Within maternity care, QI involves monitoring adverse outcomes using performance indicators to identify areas for improvement. The Adverse Outcomes Index (AOI) is a commonly used scale, in maternity QI [2].

The AOI assesses quality of labour care across providers and institutions [2]. It is a composite of 10 adverse maternal events (death, uterine rupture, Intensive Care Unit (ICU) admission, birth trauma, unanticipated operative procedures, blood transfusion, 3rd or 4th degree perineal laceration) and adverse neonatal events (death, Neonatal Intensive Care Unit (NICU) admission, 5-minute Apgar score $<7$, birth trauma) related to labour and delivery, that can be calculated using a populationbased database [2]. It is used to monitor trends in the safety of labour and delivery over time, between hospitals, and providers [2]. The AOI includes the Weighted Adverse Outcome Score (WAOS), and Severity Index (SI). The WAOS utilizes the same components, but weights the events according to their severity, while the SI describes the average degree of severity among pregnancies with an adverse event. The weighting of outcomes in the WAOS was based on expert consensus that neglected the patient voice.

The AOI addresses one aspect of the IOM's description of quality care, the safety and effectiveness of health care, but neglects another important factor identified by the IOM, patient-centred health care. The IOM defines patientcentered care as: (1) respect for patients' values, prefer- 
ences and expressed needs; (2) coordination and integration of care; (3) information, communication, and education; (4) physical comfort; (5) emotional support, relieving fear, and anxiety; and (6) involvement of family and friends [1].

Patient-centered care (PCC) has been shown to increase both patient and health care provider (HCP) satisfaction, as well as increase health care system efficiency [3]. Shared decision making is a valuable tool for maternity care providers to make their practices more patientcentered [4-6]. Shared decision making is defined as a process in which clinicians and patients collaborate to reach an evidence-informed decision congruent with patients' values [7].

The AOI provides a useful lens to evaluate policy and protocol; however, its weakness is that it was developed based on expert opinion, without the patients' perspectives. Our research goal is to develop performance indicators for labour and birth that reflect the patient perspective. That includes developing performance indicators that reflect their goals for labour and delivery, as well as revising the AOI to reflect the patient perspective. We hope to ultimately achieve these goals through a survey platform with broad patient input. The specific aims of this study, are focused on developing that platform through exploring patients' values with respect to both the labour and birth process as well as developing descriptions of complications, towards developing a survey tool that will inform development of a patient informed version of the WAOS.

\section{Materials and methods}

\subsection{Source and availability of data and material}

The data from this study was collected through a focus group and structured interviews. The data is available in anonymized format for review, by request from the corresponding author.

\subsection{Methods}

Recognizing the potential to unconsciously incorporate HCP assumptions into the study process, we undertook a multi-disciplinary, patient co-led approach to our design. Our team includes patients, midwives, obstetricians, medical trainees, a clinical research professional and a research coordinator. Research ethics approval was obtained from the Providence Health Care Research Ethics Board (H1602688).

Our ultimate goal for this research is to survey a large sample of maternity patients using a platform developed through qualitative research. For this first phase of the study, we undertook a qualitative study to investigate patient values around labour and birth using semi-structured interviews. As our main research goal was to assess how well the AOI reflects patient values, we needed to first assess patients' views on the current version of the AOI. Towards this end, we developed a version of the AOI that is accessible to patients, with the use of concise, accurate and balanced descriptions of each parameter in the index.

We developed these descriptions through an iterative process that began with a literature search of each complication to define prevalence and described short-term and long-term consequences of experiencing the complication. These descriptions were concise, accessible ( $<$ grade 8 readability), and unbiased. Draft descriptions were tested for face validity in a focus group of experts in maternity care. We enlisted the Perinatal Coordinating Council of Vancouver Coastal Health Maternal Child Program. The Council is composed of 12 interdisciplinary clinicians and administrators focusing on acute and community maternity care. The represented disciplines include family medicine, midwifery, obstetrics, and maternity nursing. The feedback from this focus group informed the next version of parameter descriptions, which were then tested on a sample of patients using semi-structured interviews.

Participants were recruited for the interviews via a convenience sample enlisted from midwifery and obstetrical clinics. To meet inclusion criteria, patients needed to be no more than 1 year from delivery and over 19 years of age. Participants were given the option of being interviewed via telephone, in-person, or by email. The purpose of the interviews was to have participants comment on the readability and comprehension of the adverse event descriptions. We also asked them to describe their expectations and experiences with their labour and birth. We asked: "What were the best and worst parts of the labour and birth?" "What were your expectations going into labour and how did those compare with your experience?"

\subsection{Analysis}

Participant responses were recorded, transcribed, coded and analyzed using Braun and Clarke's (2006) [8] reflexive approach of thematic analysis. One researcher independently reviewed the data and developed initial codes. The eventual themes were developed by organising codes with similar meanings that emerged in the data. A second researcher reviewed the data and the analysis conducted by the first researcher to ensure agreement of theme concepts and theme names.

\section{Results}

Our sample included 11 new mothers, delivered by midwives, family physicians, or obstetricians. Five themes emerged during analysis of patients' descriptions of their experiences and values pertaining to their labour and birth: (1) desire for patient-centred care, (2) improved communication, (3) labour/birth, expectations and outcomes; (4) care team support during labour and birth; (5) continuing emotional and physical postpartum care.

\subsection{Theme 1: patient-centered care}

All participants spoke of the importance of patientcentered care, including a desire to be involved in decision- 
making regarding their own labour and birth, as well as confirmation from the healthcare team that their views were considered. Participants suggest this approach to care enhances their confidence in their healthcare team. Many women felt that patient-centered care was lacking in their experience of labour and birth, with some participants describing feeling disrespected by HCPs who did not involve them in decision-making.

"My big thing about birth was being heard ... and feeling like you had a choice ... where you played a role in it and you decided what happened to you and your baby." Patient 9

"regardless of the outcome or how you get somewhere, being able to be a part of the process I think is the most-it's the most important thing for me ...it doesn't matter what mode of delivery you have or which interventions end up needing to happen but being able to be a part of the processes seems to really make... people feel confident and comfortable in what has happened." Patient 8

"When the patient doesn't feel listened to- they don't have the trust that their care is going to be safe or effective because they don't feel like the person's listening to them." Patient 7

\subsection{Theme 2: improved communication}

Participants expressed a desire for better interprovider communication and patient-provider communication during the labour and birth process. They described instances where HCPs made decisions about their care, such as a change in plan during labour, without communicating the reasons to them. Many participants also described instances where members of their care team were not communicating effectively with one another, which negatively impacted the patient's experience. Participants highlighted the importance of communication regarding care decisions that provided sufficient time for information processing. This was especially pertinent in instances where patient consent was required.

"The $O B$ consult was by far the worst thing. Um, that was him talking to my husband and not to me and him being pretty negative and not giving clear reasons for things and everything was a rush for no like- upon reflection there wasn't a reason for-for things to be a big rush." Patient 4

"They were having conversations amongst themselves and just kind of being a bit dismissive towards me." Patient 7

"I think... frequent communication - letting the parents know what's happening, why they're doing this, and what maybe the expected outcome of that would be, wouldI'm sure that would help ease the transition as well." Patient 7

\subsection{Theme 3: labour and birth expectations and outcomes}

This theme captures participants' expectations of the labour and birth process and what they prioritized as im- portant aspects of their care. All participants considered a healthy baby and healthy mother as the most important outcomes. Achieving this with a vaginal birth, and minimal intervention, was another desired outcome for many women, with cesarean section as a last resort. Access to a variety of pain management options was another important expectation of participants. They spoke of a desire to have autonomy over the uptake and type of pain management and emphasized the importance of clear communication from HCPs to the patient.

"that he was born healthy, that was my main, most important outcome. Um I was ready for any complications, I was-obviously I wouldn't have been happy but if I needed to go... but if I needed to go into hospital I was ready for, you know to do that, or ready for any interventions that my midwife suggested. I was open to whatever needed to be done. But yeah, my ultimate outcome was that he was born healthy." Patient 2

"I was hoping for a vaginal birth without any likewithout a vacuum, without forceps um and I was hoping that I didn't um need an episiotomy ... and I would get skin-toskin time and he would be breathing fine and that his heart rate was good throughout the entire birthing process and afterward." Patient 4

"...Some people would say if they wanted an epidural and they couldn't get it for whatever reason, that can be a real source of trauma." Patient 3

\subsection{Theme 4: care team support during labour \& birth}

Women highlighted the importance of physical and emotional support during labour as an integral part of their experience. Beyond trusting their care team to make evidence-based decisions and provide the best quality of care, they valued the supportive rapport with their care team. Moreover, this support came largely from a nurse or doula with several women noting that their doctor or midwife did not meet their expectations for physical and emotional support. For some women the presence of a supportive care team was the best part of their labour and birth experience, while those with less support describe more negative experiences.

"there were a few best things I think...so I had like the nurses at the hospital in the delivery room were just amazing, amazing, amazing. I felt really supported by the nurses in the hospital also my midwives were like supportive and explained things to me um pretty-pretty well um as did the nurses. I had really a lot of support through that and my doula was awesome too. I'm so happy that she was there because I think that for me to be able to push out the baby I really needed that extra support." Patient 5

"My interaction with the $O B$ and how I feel like he didn't respect or didn't really listen to what I said um or respect my opinions.... the way he interacted with me just made me feel like I was very stupid for having a different view than he did." Patient 1 
"the support of my doula and my husband was the best ... I had a midwife, but I don't remember interacting with at all, she was out of the room ..., she was not very hands on with me. And I don't know if that's because I had likelike a team of people but um I was expecting more from my midwife and I didn't really get that." Patient 4

\subsection{Theme 5: continuing emotional and physical post-partum care}

Women identified emotional vulnerability during the postpartum period, especially if they suffered complications during labour or birth. They also expressed a need for postpartum care that attends not only to postpartum physical needs, but also to emotional needs such as postpartum depression.

"I would hope that if something happens to a mother during her labour or birth that requires [an intervention], that um there is some support for her during and after to help her um you know deal with this." Patient 7

"I guess maybe a question about like, how the person felt after like, in the day and or weeks following ...the delivery like if there was an adverse outcome um how it affected them um emotionally or any other aspect like with their families or as well so any other affects from the adverse outcome that happened in the days and weeks following. " Patient 1

"maternal mental health down the road... because that's not at the time of birth, but there's definitely um I just think a lot of things result in poor maternal mental health after birth. if there was something, some way to measure the maternal like experience at the time of delivery, um would be an interesting additional thing to measure." Patient 8

In all, the patients' assessments of the AOI descriptions confirmed their readability. Several descriptions were modified at patient suggestion to improve comprehension for the final survey that will be disseminated in the second phase of the study. In addition, some patients found some descriptions were anxiety and stress provoking. To ameliorate this, we have included contact information for postpartum mental health services in our final survey.

\section{Discussion}

Our study gives voice to patient-centred values with respect to labour and delivery outcomes. The themes that arose when patients spoke of their intrapartum experiences were diverse, and not always focused on the adverse outcomes traditionally tracked as performance and quality indicators. Moreover, these adverse outcomes are not the common experience for most North American women experiencing labour and birth. While many of the participants fortunately did not experience the adverse outcomes included in the AOI, many still described some form of negative event (e.g., poor labour support, poor communication, and lack of shared-decision making) that resulted in a less than satisfying birthing experience.
Patient-centred care and clear communication were the major values conveyed by the patients in this study. Good communication and shared decision making led to patients describing their labour and birth as a satisfying experience. The converse was also true with some patients describing feeling stupid, disrespected, or dismissed by members of their health care team. Patients also described having difficulty trusting that the treatment they needed would be safe and effective when HCPs did not listen to their opinions or engage them in shared-decision making.

Patients prioritized good health outcomes for mother and child. Beyond that, many expressed a desire to deliver vaginally, with minimal interventions. Additionally, while our study was focused on intrapartum care, there was a clearly stated concern for continuing emotional and physical support during the postpartum period.

The strength of this study is that it brings the voice and values of the patients to the QI process. Our engagement with patients as co-researchers in every stage of the study brings a unique perspective that allowed us to identify and avoid biases and assumptions held by HCPs.

One limitation of this study was the utilization of multiple methods of interviewing, which specifically eliminated the opportunity to ask patients to elaborate on email responses. We included this option knowing that having a child under one year old presents significant time constraints and the email option offered an accessible interview medium. We also limited the interviews to specific research questions, without collecting specific personal demographic and clinical information. While this streamlined the interviews, it also limited our ability to assess the impact of these parameters on outcomes of interest. Other limitations of this study include the lack of generalizability that is inherent in qualitative research, as well as the selection bias that can occur with a convenience sample [9].

The global recognition of the need for respectful maternity care, and its intersection with power dynamics in the health system that can compromise the quality of maternity care, underlines the importance of defining women's aspirations for maternity care and the factors that undermine them [10]. The finding that postpartum mothers value PCC, is consistent with prior studies [3,4]. Health-care providers also identify PCC as an important strategy for improvement in health care systems [11,12]. Despite this our research shows that patients are still experiencing times where they feel unheard or even excluded from the decision-making process. In their analysis of decision making around mode of delivery following Caesarean delivery, Kaimal and Kupperman [4] noted that "despite patients and practitioners access to the same statistics, their experiential knowledge differs in quantity and quality which can result in discordant interpretations of the same evidence". This means that when faced with the same statistical probability of an adverse outcome, patients and HCPs may make different decisions. Furthermore, they acknowledge that there is a spec- 
trum of values held by patients both regarding the decisionmaking process as well as the mode of birth [4]. Our study similarly demonstrated different values and interpretation of evidence by patients and HCPs.

Our finding of patients experiencing poor communication is worth note, as perceived negative interactions with the health care system have been shown to decrease trust and may contribute to patients resisting recommendations made by their HCPs $[13,14]$. Surveys of maternal experience in the UK have found a similar gap in postnatal support [15-17]. The Canadian Maternity Experiences Survey was designed by the Public Health Agency of Canada to examine Canadian women's experiences, practices, perceptions and knowledge during pregnancy, birth and the early postpartum period [18]. While completed by women giving birth in 2006, it reflected similar themes to our work. The majority of respondents felt that their providers were competent, yet a third of women were seeking better information and more compassion from their birth attendants. Aside from this survey, there is a paucity of information about the birth experience of Canadian women, but other jurisdictions have more recent data. The National Health Service (NHS) in the United Kingdom regularly surveys mothers about their experiences during pregnancy, birth and postnatal care. The most recent survey published in 2019, showed improvements in partner support, listening to mothers and better communication with providers [19]. This survey addresses many of the issues identified as patient priorities by our study, and given its methodology and longitudinal data from prior surveys in 2013, 2015, and 2017 , it offers a proven survey methodology that could inform a patient outcome index.

\section{Conclusions}

Our finding that patients expect both a healthy mother and baby at the end of the labour and birth, highlights the need to balance outcomes for both. The identification of a perceived gap in intrapartum communication accentuates the need to better understand and embed patient values in maternity care. The current AOI is broadly utilized to track adverse outcomes but has not considered the maternity patient values. Incorporating patient values into maternity care, including the AOI, is a priority for realizing respectful maternity care. The tools developed through this study will allow us to pursue this work in the next phase of this program. The adverse outcome descriptions will inform a web-based survey that will seek to investigate the breadth of patient perspective on how these outcomes should be weighted in a patient-centered version of the AOI. The survey will also incorporate assessments of patients' perceptions of involvement in decision-making, communication between HCPs and patients/families, patient autonomy and respect, continuity of care, and post-partum support.

Further studies should determine whether the values identified in this study are shared among wider and more diverse populations. In addition, future research should examine how we can identify and support HCPs who are not engaging in patient-centered care, in order to provide education and direction. By developing an understanding of patients' values pertaining to both adverse outcomes and the labour and birth experience, we will be better prepared to counsel patients and engage in more meaningful, patientcentered care.

\section{Abbreviations \\ QI, Quality Improvement; IOM, Institute of medicine; AOI, Adverse Outcome Index; ICU, Intensive Care Unit; NICU, Neonatal Intensive Care Unit; WAOS, Weighted Adverse Outcome Score; SI, severity Index; HCP, health care provider; PCC, Patient centred care; NHS, National Health Service.}

\section{Author contributions}

GWC proposed the concept for this study, and all authors participated in the study design. LK and MS developed the initial adverse outcomes descriptions which were edited and finalized by all authors. The Focus group with the Perinatal Coordinating Council was run by GWC, NK, and LK. Participant Recruitment was performed by JN, NK, $\mathrm{KC}$, and MS. Patient interviews were done by GEJ, LK, $\mathrm{NK}$, and MS. Thematic analysis was done by CM and JN. All authors participated in the writing and finalizing of the manuscript.

\section{Ethics approval and consent to participate}

All participants provided consent to participate in this study. Research ethics approval was obtained from the Providence Health Care Research Ethics Board (H1602688), which is one of the Research Ethic Boards affiliated with the University of British Columbia. The patient consent was verbal as part of the interview, and this process was approved by the Research Ethics Board.

\section{Acknowledgment}

Thank you to all of the midwifery clinics and OBGYN practices that allowed us to recruit participants. Thank you to patients and members of the Perinatal Coordinating Council of Vancouver Coastal Health Maternal Child Program that participated in this study.

\section{Funding}

The St. Paul's Hospital Foundation provided a competitive grant to support this work in 2017, through the St. Paul's Foundation Patient Enhancement Fund (Fund number EPC \#2018-184). MS was the principal investigator and GWC the co-investigator. Because the grant was awarded as a competitive research grant, the St. Paul's Hospital Foundation had no role in the design of the study, nor the collection, analysis, interpretation of data, nor in the writing 
of the manuscript.

\section{Conflict of interest}

The authors declare no conflict of interest.

\section{Consent for publication}

Subjects provided verbal consent for recording and anonymous transcription of their interviews for research and publication purposes. The consent process did not specify the use of direct quotes to be published in this manuscript.

\section{References}

[1] Institute of Medicine, Committee on the Quality of Health Care in America. Crossing the Quality Chasm: a new health system for the 21st century. National Acadamies Press: Washington, DC. 2001.

[2] National Perinatal Information Center (NPIC). Quality Analytic Services. Special Report: The Adverse Outcome Index (AOI) update Ten Years after the Original Study. National Perinatal Information Center. 2013.

[3] Bauman AE, Fardy HJ, Harris PG. Getting it right with patient centred care. The Medical Journal of Australia. 2003; 179: $253-$ 256.

[4] Kaimal AJ, Kuppermann M. Understanding Risk, Patient and Provider Preferences, and Obstetrical Decision Making: Approach to Delivery after Cesarean. Seminars in Perinatology. 2010; 34: 331-336.

[5] Little MO, Lylerly AD, Mitchell LM, Armstrong EM, Harris, LH, Kukla, R, et al. Mode of delivery: towrd responsible inclusion of patient preference. 2009; 112: 913-918.

[6] Alsayegh E, Bos H, Campbell K, Barrett J. No.361-Cesarean delivery on maternal Request. Journal of Obstetrics and Gynaecology Canada. 2018; 40: 967-971.

[7] Grad R, Legare F, Bell NR, Dickinson JA, Singh H, Moore AE, et al. Shared decision making in preventive health care: What it is; what it is not. Canadian Family Physician. 2017; 63: 682624.
[8] Braun V, Clarke V. Using thematic analysis in psychology. Qualitative Research in Psychology. 2006; 3: 77-101.

[9] Smith J, Noble H. Bias in research. Evidence-Based Nursing. 2014; 17: 100-101.

[10] Downe S, Lawrie TA, Finlayson K, Oladapo OT. Effectiveness of respectful care policies for women using routine intrapartum services: a systematic review. Reproductive Health. 2018; 15: 23.

[11] Montague T, Gogovor A, Aylen J, Ashley L, Ahmed S, Martin $\mathrm{L}$, et al. Patient-Centred Care in Canada: Key Components and the Path Forward. Healthcare Quarterly. 2017; 20: 50-56.

[12] de Labrusse C, Ramelet A, Humphrey T, Maclennan SJ. Patientcentered Care in Maternity Services: a Critical Appraisal and Synthesis of the Literature. Women's Health Issues. 2016; 26: 100-109.

[13] Sheppard VB, Zambrana RE, O'Malley AS. Providing health care to low-income women: a matter of trust. Family Practice. 2004; 21: 484-491.

[14] Spelke B, Has P, Clark MA, Kanno L, Booker T, Werner E. Trust in physician and health agency impact postpartum choices in women with gestational diabetes. Obstetrics \& Gynecology. 2018; 131: 157S

[15] Redshaw M, Henderson J. Safely delivered: a national survey of women's experience of maternity care 2014. National Perinatology Unit: Nuffield Department of Population Health, University of Oxford. 2015.

[16] Plotkin L. Support Overdue: Women's experiences of maternity services 2017. The National Federation of Womens's Institutes: London. 2017.

[17] Cheyne H, Critchley A, Elders A, Hill D, Milburn E, Paterson A. Having a baby in Scotland 2015: listening to mothers. Health and Social Care, Scottish Government. 2015.

[18] Chalmers B, Dzakpasu S, Heaman M, Kaczorowski J. The Canadian maternity experiences survey: an overview of findings. Journal of Obstetrics and Gynaecology Canada. 2019; 30: $217-$ 228.

[19] Redshaw M, Martin CR, Savage-McGlynn E, Harrison S. Women's experiences of maternity care in England: preliminary development of a standard measure. BMC Pregnancy Childbirth. 2019; 19: 167. 